\title{
Acoustic Sources Localization in 3D Using Multiple Spherical Arrays
}

\author{
Fangzhou Wang* and Xi Pan ${ }^{\dagger}$
}

\begin{abstract}
Direction of arrival (DOA) estimation of multiple sources using sensor arrays has been widely studied in the last few decades, particularly, the spherical harmonic analysis utilizing a spherical array. Both the number of sensors on the aperture and size of the sphere can affect the estimation accuracy dramatically. However, those two factors are conflicted to each other in a single spherical array. In this paper, a multiple spherical arrays structure is proposed to provide an alternative design to the traditional single spherical array for the spherical harmonic decomposition, to obtain better localization performance. The new structure consists of several identical spheres in a given area, and the microphones are placed identically on each sphere. The spherical harmonic analysis algorithm using the new multiple array structure for the problem of multiple acoustic sources localization is presented. Simulation results show that the multiple spherical arrays can provide a more accurate direction of arrival (DOA) estimation for the multiple sources than that of a single spherical array, distinguish several adjacent sources more efficiently, and reduce the number of microphones on each sphere without decreasing its' estimation accuracy.
\end{abstract}

Keywords: Multiple sources localization, Spherical harmonic analysis, Multiple spherical arrays

\section{Introduction}

Acoustic source localization is a passive positioning method that can be applied to target detection. The time difference of arrival (TDOA) is the usual method used in acoustic source localization, which estimates the source position using the information on the geometry $[1,2]$. The TDOA at the sensors on the geometry is a function of source direction, so the TDOA at the peaks of the crosscorrelation function can therefore be used to identify the source direction of arrival. Although the algorithm is easy to carry out, it can only deal with a single sound source at a given time [3].

The high-resolution subspace techniques, Multiple Signal Classification (MUSIC) [4-6] and Estimation of Signal Parameters via Rotational Invariance Techniques (ESPRIT) [7-9], are widely used in direction of arrival (DOA) in the past few decades. They can resolve the multiple sources localization problem [10]. Conventionally, various linear [11], circular [12] and spherical sensor arrays [13-15] are used for DOA estimation under free field propagation conditions between the source and sensors. Especially, benefiting from the three-dimensional symmetry, spherical microphone arrays offer an ideal tool for capturing and analyzing three dimensional sound fields. To get better performance, spherical harmonic analysis considering the physical characteristics of wave propagation in the air is applied to MUSIC and ESPRIT

$\dagger$ Corresponding Author: School of Mechatronical Engineering, Beijing Institute of Technology, Beijing, 100081, China. (panxi@bit.edu.cn)

* Dept. of Electrical and Computer Engineering, Stevens Institute of Technology, Hoboken, NJ 07307 USA. (fwang11@stevens.edu)

Received: June 19, 2014; Accepted: December 16, 2015 algorithms based on spherical microphone arrays [16]. The sound field is decomposed into spherical harmonic components by sampling the field using spherical harmonic analysis technique $[17,18]$. One of the main advantages of performing the analysis in the spherical harmonic component domain is the fact that the frequency-dependent components are decoupled from the angular-dependent components which provides a new perspective on related array processing problems such as source localization and detection. And owing to the efficient algorithm, the spherical harmonic analysis is more practical [19]. Teutsch and Kellermann $[3,20]$ proposed the "eigenbeams" (EBs)ESPRIT localization method for multiple wideband acoustic sources and the "eigenbeams" is actually the spherical harmonic. In [21], the spherical harmonic based MUSIC algorithm (SH-MUSIC), where the spherical harmonic transformation is operated before the MUSIC algorithm, is used to do the estimation.

The aim of our study is to improve the performance of SHMUSIC algorithm by using a new microphone array. Notice that when using the spherical harmonic analysis techniques, the microphones of the traditional array are arranged regularly, such as circular array or spherical array $[18,22-24]$. More importantly, only single array is used in all the former related study [25-28]. In our application, the microphones on each sphere are distributed regularly, but a set of spheres are placed on a plane, aiming at obtaining higher accuracy and efficiency of estimation. In this multiple spheres structure, each sphere has its own coordinate, and a globe coordinate is built up to take account the phase difference among microphones from different spheres. This multiple spherical arrays (MSA) can not only meet the randomly placing requirement of spheres 
but also reduce the number of microphones on each sphere which is restricted by the finite spherical region when using single spherical array. Besides, an improved SHMUSIC algorithm for multiple spherical arrays is discussed. The simulation results based on the novel structure verify our claim.

The remainder of this paper is organized as follows. In Section 2, we introduce the signal model and the spherical harmonic decomposition of sound field. Section 3 briefly discusses the simplified localization algorithm using spherical harmonic analysis. The localization method using multiple spherical arrays is discussed in section 4 and the simulation examples are presented in Section 5. Finally, the conclusions are drawn in Section 6.

\section{Signal model and Problem Formulation}

\subsection{Sound field}

Assume that all the waves applied in this paper are plane waves, the model of two planar waves impinging on a spherical aperture from two far field sources $S 1$ and $S 2$ is shown in Fig. 1.

Supposing that two plane waves with unit magnitude impinge on a sphere from directions $\left(\theta_{1}, \phi_{1}\right)$ and $\left(\theta_{2}, \phi_{2}\right)$, so the incident field $P_{1}(r, \vartheta, \varphi)$ at the observe point on the sphere surface can be expressed as [16, 29]:

$$
\begin{aligned}
& P_{1}(r, \vartheta, \varphi)=w_{1} e^{i \mathbf{k}_{1} \cdot \mathbf{r}}+w_{2} e^{i \mathbf{k}_{2} \bullet \mathbf{r}} \\
& =w_{1} \sum_{n=0}^{\infty} \sum_{m=-n}^{m=n} 4 \pi(i)^{n} b_{n}(k r) Y_{n m}(\vartheta, \varphi) Y_{n m}^{*}\left(\theta_{1}, \phi_{1}\right) \\
& +w_{2} \sum_{n=0}^{\infty} \sum_{m=-n}^{m=n} 4 \pi(i)^{n} b_{n}(k r) Y_{n m}(\vartheta, \varphi) Y_{n m}^{*}\left(\theta_{2}, \phi_{2}\right) \\
& =\sum \sum 4 \pi(i)^{n} b_{n}(k r) Y_{n m}(\vartheta, \varphi)\left(w_{1} Y_{n m}^{*}\left(\theta_{1}, \varphi_{1}\right)\right. \\
& \left.+w_{1} Y_{n m}^{*}\left(\theta_{1}, \varphi_{1}\right)\right)
\end{aligned}
$$

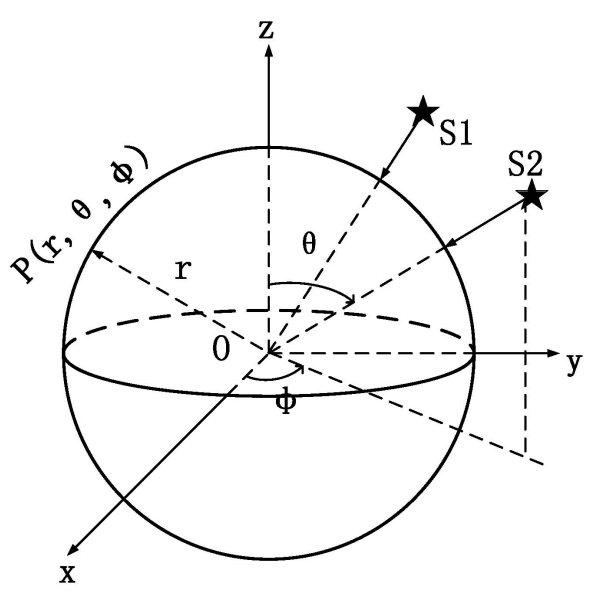

Fig. 1. Geometry of two planar waves impinging on a spherical aperture. where $k_{1}$ and $k_{2}$ are vectors pointing to the directions of the $1^{\text {st }}$ and $2^{\text {nd }}$ source $\left(\theta_{1}, \phi_{1}\right)$ and $\left(\theta_{2}, \phi_{2}\right)$ respectively, and $\left|k_{1}\right|=k_{1},\left|k_{2}\right|=k_{2}$, with $k_{1}$ and $k_{2}$ are the wavenumber of $1^{\text {st }}$ and $2^{\text {nd }}$ sources, $r$ is a vector describing the location of the observe point, and $w_{1}$ and $w_{2}$ are the weights of the sources. $b_{n}(k r)$ is [30]:

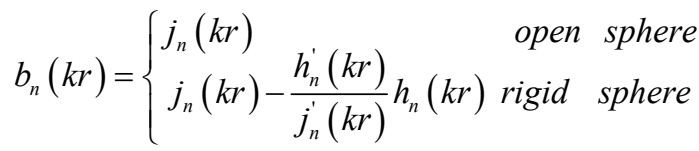

where $j_{n}(k r)$ is the spherical Bessel function and $h_{n}(k r)$ is the spherical Hankel function. In this paper, we only consider open spheres. ' $*$ ' denote complex conjugation, $Y_{n m}$ is the spherical harmonic of order $n$ and degree $m$ :

$$
Y_{n m}(\vartheta, \varphi)=\sqrt{\frac{2 n+1}{4 \pi} \frac{(n-|m|) !}{(n+|m|) !}} P_{n|m|}(\cos \vartheta) e^{j m \varphi}
$$

where $P_{n|m|}$ is the associated Legendre function. In practical application, the number of terms in (1) cannot be infinite that we can replace it with a finite $N$ which is mainly determined by the size of the sphere [31].

For $S$ sources, (1) can be rewritten as:

$$
\begin{aligned}
& P_{1}(r, \vartheta, \varphi)=\sum_{s=1}^{S} w_{s} e^{i \mathbf{k}_{\mathrm{s}} \bullet \mathbf{r}}=\sum_{n=0}^{\infty} \sum_{m=-n}^{m=n} 4 \pi(i)^{n} \\
& \times\left(\sum_{s=1}^{S} w_{s} b_{n}(k r) Y_{n m}(\vartheta, \varphi) Y_{n m}^{*}\left(\theta_{s}, \phi_{s}\right)\right)
\end{aligned}
$$

Where $w_{s}$ and $k_{s}$ are the weight and direction vector of the $s^{\text {th }}$ source.

\subsection{Sound field decomposition}

Spherical harmonic describes an orthonormal decomposition of the pressure of sound field, and the core of spherical harmonic decomposition is the forward and inverse spherical fourier transform. Assume a spherical coordinate, there is a function $f(\vartheta, \varphi)$ which is square integrable on the unit sphere, then the spherical fourier transform of $f$, denoted by the fourier coefficient $f_{n m}$, is given by [32]:

$$
\begin{gathered}
f(\vartheta, \varphi)=\sum_{n=0}^{\infty} \sum_{m=-n}^{m=n} f_{n m} Y_{n m}(\vartheta, \varphi) \\
f_{n m}=\int_{0}^{2 \pi} \int_{0}^{\pi} f(\vartheta, \varphi) Y_{n m}^{*}(\vartheta, \varphi) \sin \vartheta d \vartheta d \varphi
\end{gathered}
$$

where (5) and (6) represent the forward and inverse spherical fourier transform, respectively. $Y_{n m}$ is the spherical harmonic of order $n$ and degree $m$. We define a 
decomposition coefficient $a_{n m}(k)$ which is expressed as:

$$
a_{n m}(k)=4 \pi(i)^{n} b_{n}(k r) Y_{n m}^{*}(\theta, \phi)
$$

and shows that the coefficient is independent from the radial and angular information of the observer points. Combining (1) and (7), the sound field at the point $P_{l}(k r, \vartheta, \varphi)$ can also be expressed in terms of spherical harmonic expansion as:

$$
P_{1}(r, \vartheta, \varphi)=\sum_{n=0}^{\infty} \sum_{m=-n}^{m=n} a_{n m}(k) Y_{n m}(\vartheta, \varphi)
$$

According to the spherical fourier transform introduced above, (8) can be transformed to:

$$
a_{n m}(k)=\int_{0}^{2 \pi} \int_{0}^{\pi} P_{1}(r, \vartheta, \varphi) Y_{n m}^{*}(\vartheta, \varphi) \sin \vartheta d \vartheta d \varphi
$$

In practical application, the spherical integration cannot be worked out, thus $P_{l}(k r, \vartheta, \varphi)$ is measured by the spherical microphone array. Considering a sphere with $T \times H$ uniformly distributed microphones, then (9) can be rewritten as:

$$
\hat{a}_{n m}(k)=\sum_{t=1}^{T \times H} \alpha_{t} P_{1}\left(r, \vartheta_{t}, \varphi_{t}\right) Y_{n m}^{*}\left(\vartheta_{t}, \varphi_{t}\right)
$$

where $\alpha_{t}$ is the coefficient to make sure the accuracy of approximation from the integral to the summation, and $\alpha_{t}$ can be expressed in the form of:

$$
\alpha_{t}=\sin \vartheta_{t} \Delta \vartheta \Delta \varphi
$$

where $\Delta \vartheta=\pi / T, \Delta \varphi=2 \pi / H$.

\section{Multiple Sources Localization Algorithm}

Considering $S$ sources with directions $\left(\theta_{l}, \phi_{l}\right)$ $(l=1,2, \cdots, L)$, the decomposition coefficient of multiple sources can be obtained based on (4) and (10) :

$$
\hat{a}_{n m}(k)=\sum_{s=1}^{S} \sum_{t=1}^{T \times H} w_{s} e^{i \mathbf{k}_{\mathbf{s}} \cdot \mathbf{r}} Y_{n m}^{*}\left(\vartheta_{t}, \varphi_{t}\right) \sin \vartheta_{t} \Delta \vartheta \Delta \varphi
$$

Suppose M observe points with directions $\left(\theta_{l}, \phi_{l}\right)$ $(l=1,2, \cdots, L)$ on the sphere, we can express the decomposition coefficient as matrix forms: $p_{n m}=$ $B \times C \times W$, where

$$
\mathbf{p}_{n m}=\left[\hat{a}_{00}, \hat{a}_{1-1}, \hat{a}_{10}, \hat{a}_{11}, \cdots, \hat{a}_{N N}\right]^{T}
$$

$p_{n m}$ is a $(N+1)^{2} \times 1$ matrix.

$$
W=\left[w_{1}, w_{2}, \cdots, w_{S}\right]^{T}
$$

and $C$ is an $M \times S$ matrix:

$$
C=\left[\begin{array}{cccc}
\sin \vartheta_{\vartheta} e^{i \mathbf{k}_{1} \bullet \boldsymbol{r}_{1}} & \sin \vartheta_{1} e^{i \mathbf{k}_{2} \bullet \boldsymbol{r}_{1}} & \cdots & \sin \vartheta_{1} e^{i \mathbf{k}_{s} \bullet \boldsymbol{r}_{1}} \\
\sin \vartheta_{2} e^{i \mathbf{k}_{1} \bullet \boldsymbol{r}_{2}} & \sin \vartheta_{2} e^{i \mathbf{k}_{2} \bullet \boldsymbol{r}_{2}} & \cdots & \sin \vartheta_{2} e^{i \mathbf{k}_{\boldsymbol{s}} \bullet \boldsymbol{r}_{2}} \\
\vdots & \vdots & \vdots & \vdots \\
\sin \vartheta_{M} e^{i \mathbf{k}_{\mathbf{1}} \bullet \boldsymbol{r}_{M}} & \sin \vartheta_{M} e^{i \mathbf{k}_{2} \cdot \boldsymbol{r}_{M}} & \cdots & \sin \vartheta_{M} e^{i \mathbf{k}_{\mathbf{s}} \bullet \boldsymbol{r}_{M}}
\end{array}\right]
$$

And $B=(\Delta \vartheta \Delta \varphi) \times Y$, where $Y$ is a $(N+1)^{2} \times M$ matrix:

$$
\mathbf{Y}=\left[\begin{array}{ccccc}
Y_{00}^{*}\left(\vartheta_{1}, \varphi_{1}\right) & Y_{00}^{*}\left(\vartheta_{2}, \varphi_{2}\right) & \cdots & Y_{00}^{*}\left(\vartheta_{M}, \varphi_{M}\right) \\
Y_{1-1}^{*}\left(\vartheta_{1}, \varphi_{1}\right) & Y_{1-1}^{*}\left(\vartheta_{2}, \varphi_{2}\right) & \cdots & Y_{1-1}^{*}\left(\vartheta_{M}, \varphi_{M}\right) \\
Y_{10}^{*}\left(\vartheta_{1}, \varphi_{1}\right) & Y_{10}^{*}\left(\vartheta_{2}, \varphi_{2}\right) & \cdots & Y_{10}^{*}\left(\vartheta_{M}, \varphi_{M}\right) \\
Y_{11}^{*} \vartheta_{1}, \varphi_{1} & Y_{11}^{*}\left(\vartheta_{2}, \varphi_{2}\right) & \cdots & Y_{11}^{*}\left(\vartheta_{M}, \varphi_{M}\right) \\
\vdots & \vdots & \vdots & \vdots \\
Y_{N N}^{*}\left(\vartheta_{1}, \varphi_{1}\right) & Y_{N N}^{*}\left(\vartheta_{2}, \varphi_{2}\right) & \cdots & Y_{N N}^{*}\left(\vartheta_{M}, \varphi_{M}\right)
\end{array}\right]
$$

Assume $A=B \times C$, then (13) can be simplified to

$$
p_{n m}=\mathbf{A} \times \mathbf{W}
$$

where A is a $(N+1)^{2} \times S$ steering matrix, $W$ is the receival signal array. Now we can use $p_{n m}$ to do the DOA estimation using MUSIC algorithm through the following processes. First, we can get the correlation matrix:

$$
\mathbf{R}_{n m}=E\left\{p_{n m} p_{n m}^{H}\right\}
$$

In practical application, suppose there are $K$ samples, so $R_{n m}$ can be estimated as:

$$
\widehat{\mathbf{R}}_{n m}=\frac{1}{K} \sum_{k=1}^{K} p_{n m}(k) p_{n m}^{H}(k)
$$

Taking eigenvalue decomposition of $\hat{R}_{n m}$ :

$$
\operatorname{eigen}\left(\hat{R}_{n m}\right)=\left[E_{a}, E_{n}\right]
$$

where $E_{a}$ and $E_{n}$ are the signal subspace and noise subspace respectively. Then we can get the spectrum equation respect to the direction of the source:

$$
\operatorname{spectrum}(\theta, \phi)=\frac{1}{a^{T}\left[E_{n} E_{n}^{*}\right] a}
$$

Where $a$ is the steering vector which is a column of $A$ for any direction. By scanning $(\theta, \phi)$, the peaks of the spectrum will be in conformity to the signals' directions. 


\section{Multiple Spherical Arrays}

Giving the spherical harmonic up to $\operatorname{order} N$, the maximum source number that the single spherical array can deal with is $N-1$. The more sources there are, the more microphones the array need. However, there are geometry limitations for single spherical array. In this paper, a multiple spherical arrays structure is proposed to estimate the directions of multiple sources.

Assuming that there are L irregularly placed spheres of radius $r$ with the same uniform distribution of microphones on each of them, while their self-coordinates are located as a circle of radius $R$ on a plane as shown in Fig. 2. Suppose $M=T \times H$ microphones on each sphere.

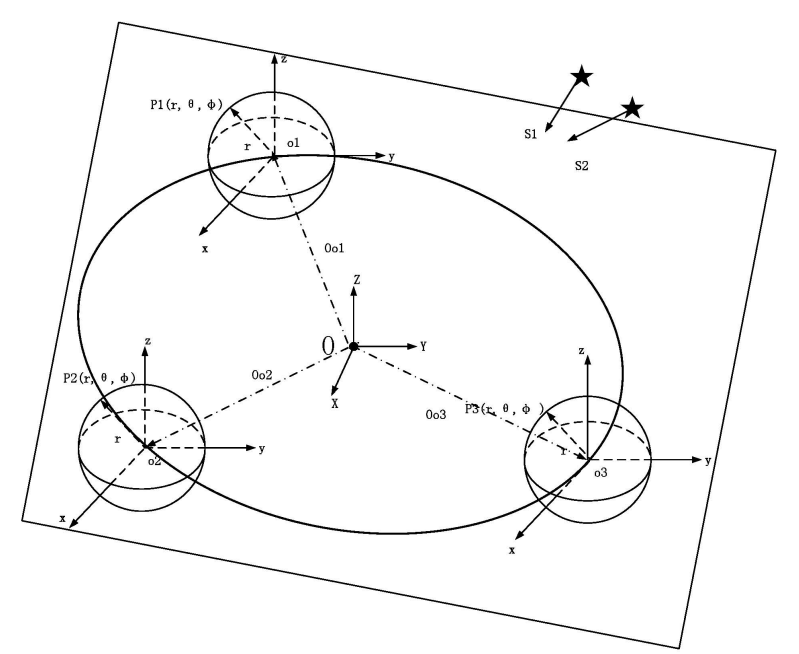

Fig. 2. Geometry of three spherical arrays

We can rewrite (10) and (4) in the following forms respectively:

$$
\begin{gathered}
\hat{a}_{n m}^{(l)}=\sum_{t=1}^{M} P_{(l)}\left(\vartheta_{t}, \varphi_{t}\right) Y_{n m}^{(l) *}\left(\vartheta_{t}, \varphi_{t}\right) \sin \vartheta_{t} \Delta \vartheta \Delta \varphi \\
P_{(l)}\left(\vartheta_{t}, \varphi_{t}\right)=\sum_{s=1}^{S} w_{s} e^{i k\left(\mathbf{r}_{o l}+\mathbf{r}_{t}\right) \cdot \hat{\mathbf{y}}_{\mathbf{s}}}
\end{gathered}
$$

where $P_{(l)}\left(\vartheta_{t}, \varphi_{t}\right)$ is the measured pressure on the $l^{t h}$ sphere at point $\left(\vartheta_{t}, \varphi_{t}\right), w_{s}$ is the receival signal of the $s^{\text {th }}$ source, $k=2 \pi f / c$ with frequency $f$ and $c$ is the speed of sound, and $\hat{y}_{s}$ is the direction vector respect to the $s^{\text {th }}$ source. $r_{o l}$ is the direction vector from the origin of the coordinate to the centre of the $l^{\text {th }}$ sphere while $r_{t}$ is the direction vector from the centre of the $l^{\text {th }}$ sphere to the $t^{\text {th }}$ microphone on the sphere $\left(\left|r_{o l}\right|=R,\left|r_{t}\right|=r\right)$. By substituting (21) into (20) we can get:

$$
\hat{a}_{n m}^{(l)}=\sum_{s=1}^{S} w_{s} \sum_{t=1}^{M} e^{i k\left(\mathbf{r}_{o l}+\mathbf{r}_{t}\right) \cdot \hat{\mathbf{y}}_{s}} Y_{n m}^{(l) *}\left(\vartheta_{t}, \varphi_{t}\right) \sin \vartheta_{t} \Delta \vartheta \Delta \varphi
$$

Also it can be expressed as:

$$
\begin{aligned}
p_{n m}^{L} & =\left[\hat{a}_{n m}^{1}, \hat{a}_{n m}^{2}, \cdots, \hat{a}_{n m}^{l}, \cdots, \hat{a}_{n m}^{L},\right]^{T} \\
& =\left[B \times C^{(1)}, \cdots, B \times C^{(l)}, \cdots, B \times C^{(L)}\right]^{T}
\end{aligned}
$$

where $p_{n m}^{L}$ is the decomposition coefficients for multiple spherical array, $B$ and $W$ has the same structure as single spherical array, and $C^{(l)}$ is an $M \times S$ matrix given by

$$
C^{(l)}=\left[\begin{array}{ccc}
\sin \vartheta_{1} e^{i k\left(r_{o l}+r_{1}\right) \cdot \hat{y}_{l}} & \cdots & \sin \vartheta_{1} e^{i k\left(r_{o l}+r_{1}\right) \cdot \hat{y}_{S}} \\
\sin \vartheta_{2} e^{i k\left(r_{o l}+r_{2}\right) \cdot \hat{y}_{l}} & \cdots & \sin \vartheta_{2} e^{i k\left(r_{o l}+r_{2}\right) \cdot \hat{y}_{S}} \\
\vdots & \ddots & \vdots \\
\sin \vartheta_{M} e^{i k\left(r_{o l}+r_{M}\right) \cdot \hat{y}_{l}} & \cdots & \sin \vartheta_{M} e^{i k\left(r_{o l}+r_{M}\right) \cdot \hat{y}_{S}}
\end{array}\right]
$$
(23):

Using matrix $A^{L}$ to substitute the $L(N+1)^{2} \times M$ matrix in

$$
A^{L}=\left[B \times C^{(1)}, \cdots, B \times C^{(l)}, \cdots, B \times C^{(L)}\right]^{T}
$$

$A^{L}$ is steering matrix of multiple spherical array, (23) can be simplified as:

$$
p_{n m}^{L}=A^{L} \times W
$$

Then, according to (15)-(19), we can find the direction of the sources using multiple spherical arrays.

\section{Simulations}

In this section, single spherical array (SSA) and multiple spherical arrays (MSA) simulations are provided to illustrate the efficiency of our methods. For all the following simulations, the comparison of performance is given in terms of root mean square error (RMSE) (averaged over the sources).

$$
R M S E=\sqrt{\frac{\sum_{s=1}^{S} \Delta \Omega_{s}^{2}}{S}}
$$

\subsection{Single and multiple arrays}

In this subsection, two incoherent sound signals $\left(S_{1}, S_{2}\right)$ impinge from approximate directions $(\theta, \phi)=\left(120^{\circ}\right.$, $\left.120^{\circ}\right)$ and $\left(270^{\circ}, 60^{\circ}\right)$, respectively, their frequency range from $0.8 \mathrm{kHz}$ to $1 \mathrm{kHz}$. Assuming that $k r=1.75$ and the ratio of signal to noise (SNR) for the two sound signals are both $0 \mathrm{~dB}$.

Twenty microphones are placed on a sphere of radius 0.1 $m$, six microphones are distributed on each latitude and two microphones on the two poles as shown in Fig. 3. 


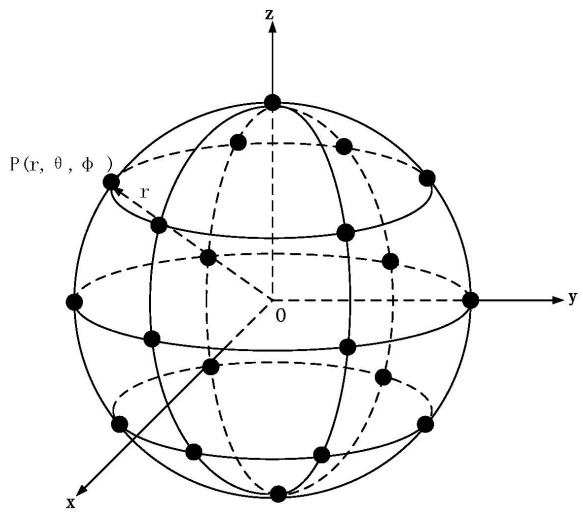

Fig. 3. Example of single spherical array structure

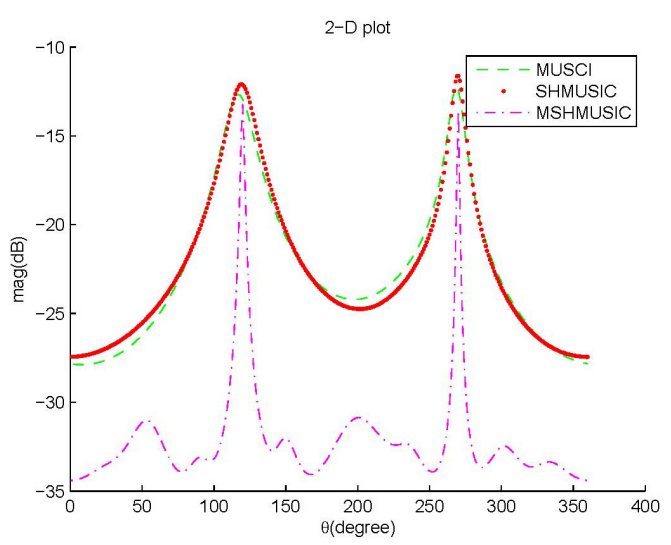

Fig. 4. DOA estimation using different algorithm and structures

As for the multiple spherical arrays model, three spheres are placed as a circle of radius $0.5 \mathrm{~m}$ on a limited plane as Fig. 2, twenty microphones are distributed on each sphere with the same arrangement as single spherical array. When the pitch angle $\phi$ of the two sources are fixed at $120^{\circ}$ and $60^{\circ}$, scanning the azimuth angle $\theta$ of them can obtain their spectrum in this direction. Fig. 4 shows the DOA estimation result for open sphere using single and multiple arrays (MUSIC in Fig. 4 means: single spherical array using MUSIC algorithm; SHMUSIC means: single spherical array using SHMUSIC algorithm; MSHMUSIC means: multiple spherical arrays using SH-MUSIC algorithm). The sidelobe in the third simulation is lower than that of the first two, through which we can distinguish the direction of sources more accurately. The performances of single spherical array and multiple spherical arrays using SH-MUSIC are shown in Fig. 5 and Fig. 6, respectively. The spectrum of multiple arrays has more pointed peak which can improve the estimation accuracy efficiently.

Making the SNR of the two sources range from $-10 \mathrm{~dB}$ to $10 \mathrm{~dB}$ simultaneously. For different SNR, simulations are operated of 200 times in order to get RMSE and estimation probability. As Fig. 7 shows, when the SNR increases, the RMSE decreases, and the multiple arrays has

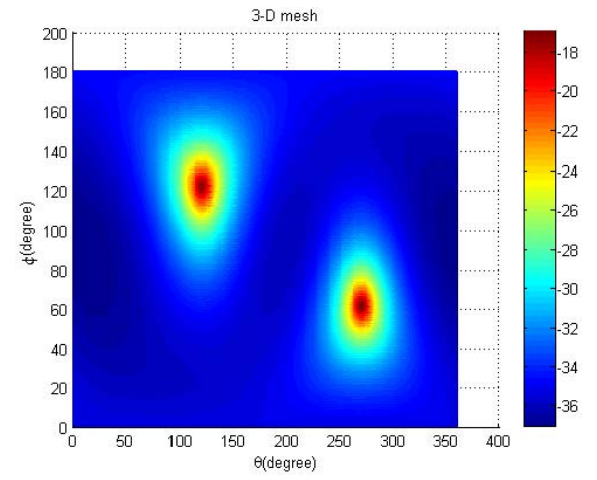

(a) Top view

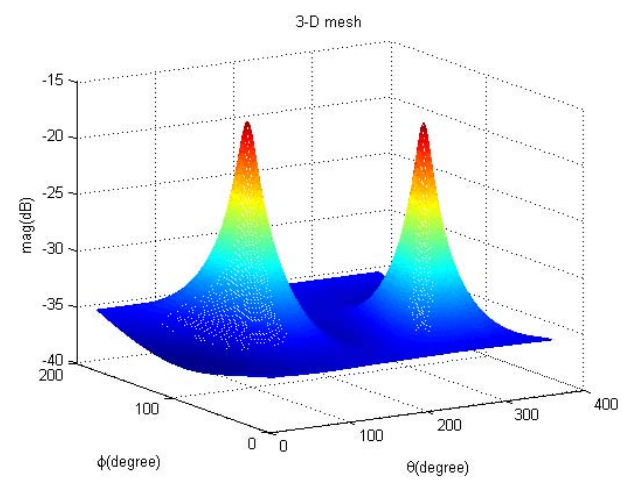

(b) Horizontal view

Fig. 5. 3-D spectrum of DOA estimation using single spherical array

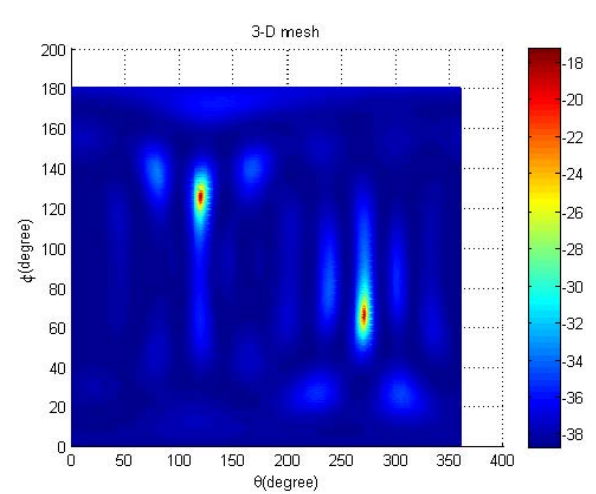

(a) Top view

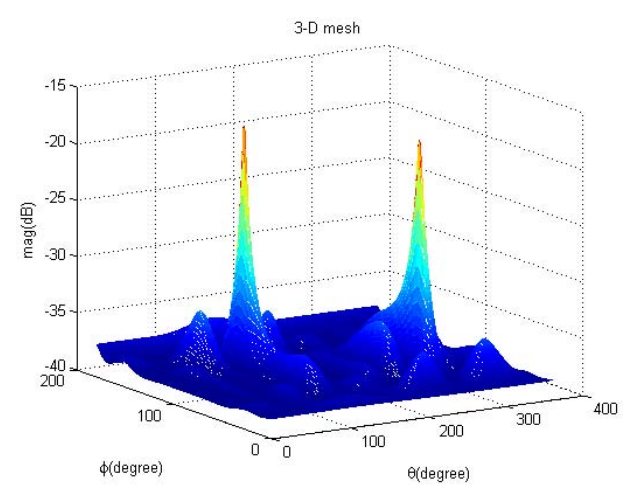

(b) Horizontal view

Fig. 6. 3-D spectrum of DOA estimation using multiple spherical arrays 


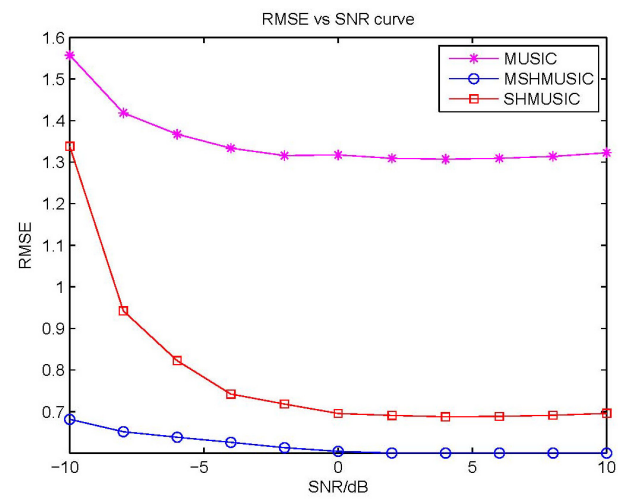

Fig. 7. RMSE of three algorithms under different SNR values

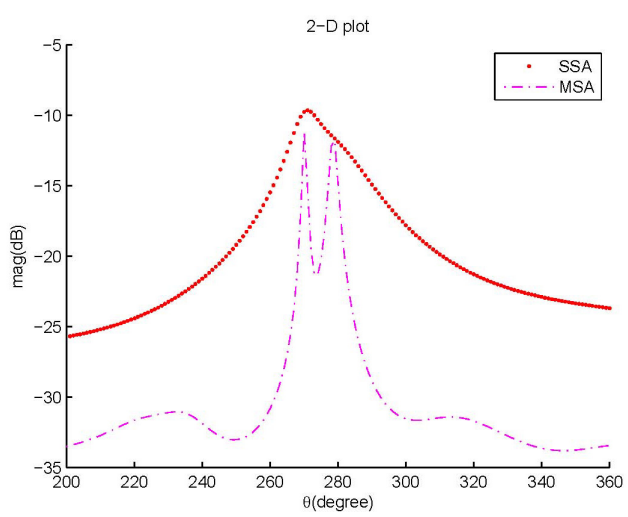

Fig. 8. Spectrums of two adjacent sources using two structures

lower RMSE than that of the single one at the same SNR, which can lead to better estimation performance in noisy environment.

\subsection{Adjacent Sources}

The lower sidelobe of the multiple spherical arrays model makes it much more effective to locate two adjacent sources than the single spherical array model as Fig. 8 shows. In this subsection, the single spherical array (SSA) model and multiple spherical arrays(MSA) model are the same as subsection 5.1. The true angles for the two sources are $270^{\circ}$ and $279^{\circ}$ respectively. The single spherical array model cannot distinguish the two sources while the multiple spherical arrays model can locate them accurately. In another multiple sources case, DOA estimation of five sound sources with direction $\left(126^{\circ}, 60^{\circ}\right)$, $\left(132^{\circ}, 66^{\circ}\right),\left(114^{\circ}, 54^{\circ}\right),\left(138^{\circ}, 48^{\circ}\right),\left(108^{\circ}, 72^{\circ}\right)$ was shown in Fig. 9.

\subsection{Various number of array}

All of the previous comparative simulations between the single and multiple arrays were based on the fact that the distributions of microphones on each sphere are the same, so the total number of microphone in those two structures

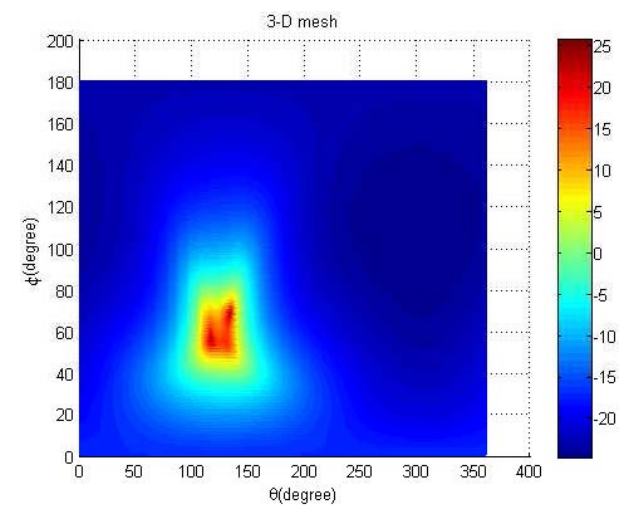

(a) single spherical array

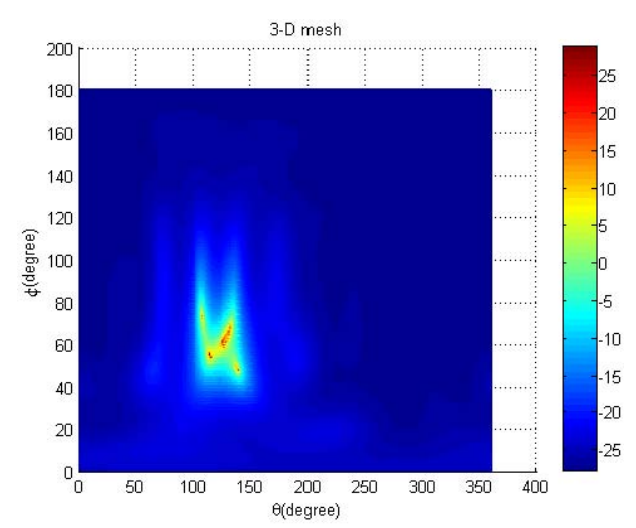

(b) Multiple spherical arrays

Fig. 9. DOA estimation of five adjacent sources

Table 1. Multiple structures

\begin{tabular}{c|c|c}
\hline & Number of spheres & Distribution $^{\mathrm{a}}$ \\
\hline Structure 1 & 1 & $5^{*} 36^{\mathrm{b}}$ \\
\hline Structure 2 & 2 & $5^{*} 18$ \\
\hline Structure 3 & 3 & $5^{*} 12$ \\
\hline Structure 4 & 4 & $5^{* 9}$ \\
\hline
\end{tabular}

a Distribution indicates the distribution of microphone on each sphere.

$\mathrm{b}$ For $\mathrm{a} * \mathrm{~b}$, a depicts the five latitude on the sphere while $\mathrm{b}$ indicates the number of microphones on each latitude.

are not equal. In this part, four structures are designed, and the total number of microphone for each structure are equal, 180 microphones in every structure. Each structure consists various number of spheres of radius $0.1 \mathrm{~m}$. The distributions of microphones on each sphere are vary from structure to structure, while the distributions are identical within the same structure. Table 1 shows the microphone distribution of four structures used in this subsection. The distribution of microphones on each latitude is referred to as equalangle distribution. Assuming six sound sources with frequency range of $(0.5 \mathrm{kHz}-1 \mathrm{kHz})$, and their directions are $\left(90^{\circ}, 30^{\circ}\right),\left(270^{\circ}, 60^{\circ}\right),\left(60^{\circ}, 150^{\circ}\right),\left(180^{\circ}, 90^{\circ}\right)$, $\left(240^{\circ}, 45^{\circ}\right)$ and $\left(300^{\circ}, 120^{\circ}\right)$, respectively. Supposing that $k r=1.67$ and the (SNR) for all the six sound signals are 0 dB. Fig. 10 shows the estimation results for all four structures. The performance of those structures under 
different SNR values is shown in Fig. 11. The single spherical array can only locate those sources vaguely, while the multiple arrays can obtain their locations accurately even when the total number of microphone for each structures are equal. The estimation of single array has such huge error that the SNR can hardly affect its RMSE, and the performance of multiple arrays can be affected by the SNR. When the SNR increases, the RMSE decrease. Although structure 4 has the same amount of microphone as other structures, this one makes all the four spheres separate more far away from each other,

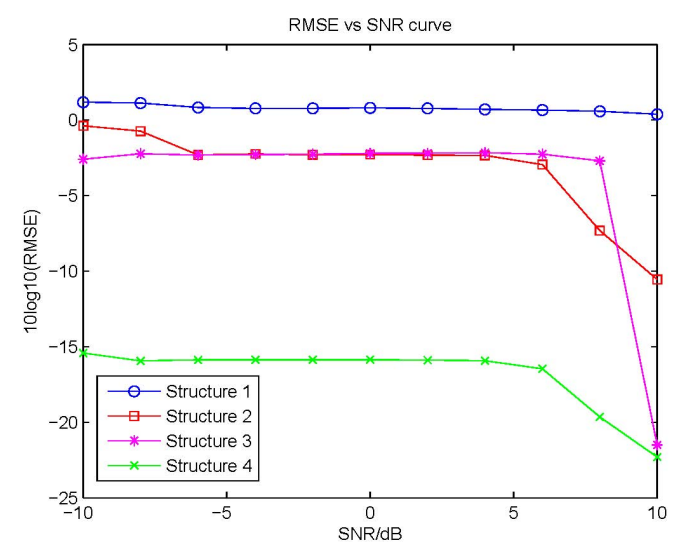

Fig. 10. RMSE of four structures under different SNR

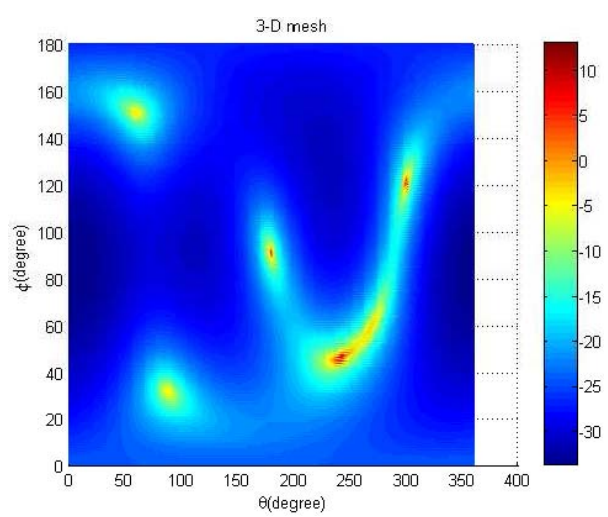

(a) Structure 1

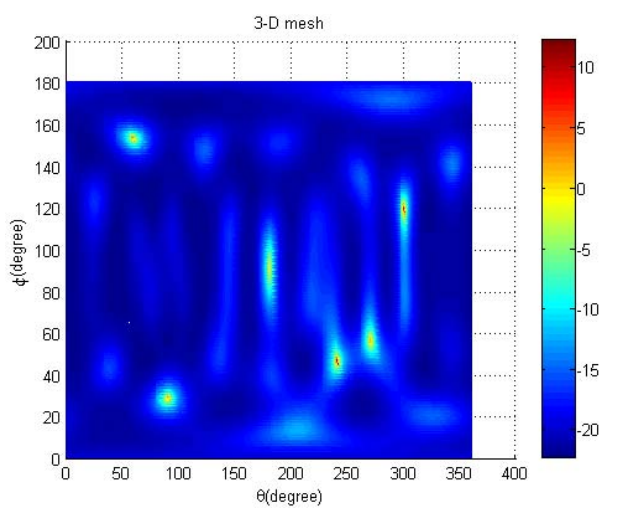

(c) Structure 3 which will provide with increased resolution than fewer spherical arrays due to spatial diversity. As shown in (1), the $k r$ value will affect the response of Bessel function. When the bandwidth of the signal is fixed, increasing $R$ will make the modal response of Bessel function more far

Table 2. Direction of sources

\begin{tabular}{ccc}
5 sources & 10 sources & 15 sources \\
$\left(90^{\circ}, 30^{\circ}\right)$ & $\left(300^{\circ}, 30^{\circ}\right)$ & $\left(150^{\circ}, 60^{\circ}\right)$ \\
$\left(270^{\circ}, 60^{\circ}\right)$ & $\left(30^{\circ}, 150^{\circ}\right)$ & $\left(150^{\circ}, 30^{\circ}\right)$ \\
$\left(60^{\circ}, 150^{\circ}\right)$ & $\left(60^{\circ}, 60^{\circ}\right)$ & $\left(60^{\circ}, 60^{\circ}\right)$ \\
$\left(240^{\circ}, 45^{\circ}\right)$ & $\left(180^{\circ}, 150^{\circ}\right)$ & $\left(30^{\circ}, 150^{\circ}\right)$ \\
$\left(180^{\circ}, 90^{\circ}\right)$ & $\left(240^{\circ}, 120^{\circ}\right)$ & $\left(120^{\circ}, 120^{\circ}\right)$ \\
& $\left(120^{\circ}, 120^{\circ}\right)$ & $\left(270^{\circ}, 60^{\circ}\right)$ \\
& $\left(270^{\circ}, 60^{\circ}\right)$ & $\left(180^{\circ}, 150^{\circ}\right)$ \\
& $\left(60^{\circ}, 120^{\circ}\right)$ & $\left(30^{\circ}, 30^{\circ}\right)$ \\
& $\left(330^{\circ}, 150^{\circ}\right)$ & $\left(300^{\circ}, 120^{\circ}\right)$ \\
& $\left(150^{\circ}, 60^{\circ}\right)$ & $\left(180^{\circ}, 60^{\circ}\right)$ \\
& & $\left(330^{\circ}, 60^{\circ}\right)$ \\
& & $\left(240^{\circ}, 120^{\circ}\right)$ \\
& & $\left(330^{\circ}, 150^{\circ}\right)$ \\
& & $\left(60^{\circ}, 120^{\circ}\right)$ \\
& & $\left(300^{\circ}, 30^{\circ}\right)$ \\
\hline
\end{tabular}

a 1 degree $=\pi / 180$ radians

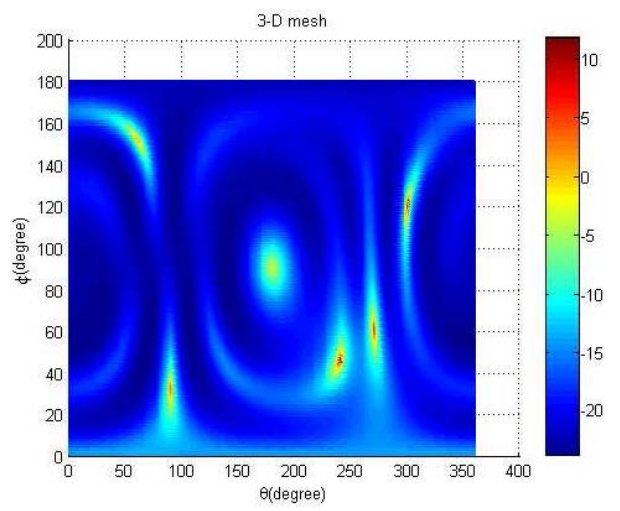

(b) Structure 2

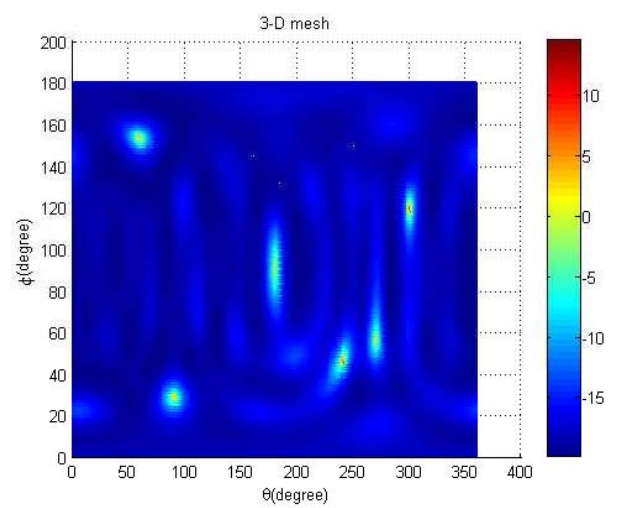

(d) Structure 4

Fig. 11. DOA estimation of four structures 
away from its zeros, which will give higher resolution. H.Teutsch's book [33] has very detailed explanation about this problem.

\subsection{Various number of sources}

For the simulations in this subsection, structure 4 described in subsection 5.3 was used. The frequency of the sources range from $0.5 \mathrm{kHz}$ to $1 \mathrm{kHz}$, and $k r=1.67$, $\mathrm{SNR}=0 \mathrm{~dB}$. Table 2 indicates the direction of those sources.

Fig. 12 depicts the estimation performance with 5,10

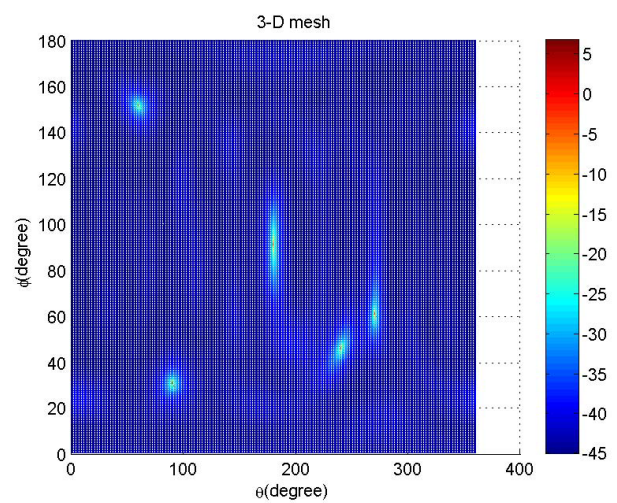

(a) 5 sources

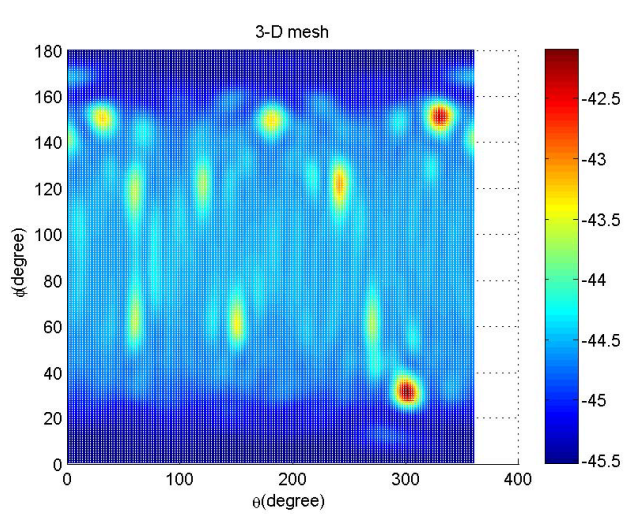

(b) 10 sources

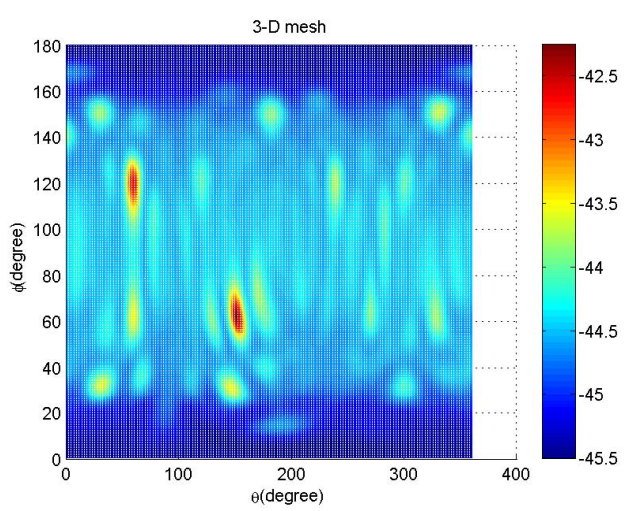

(c) 15 sources

Fig. 12. DOA estimation of multiple sources, and the position of sources are given in Table 2

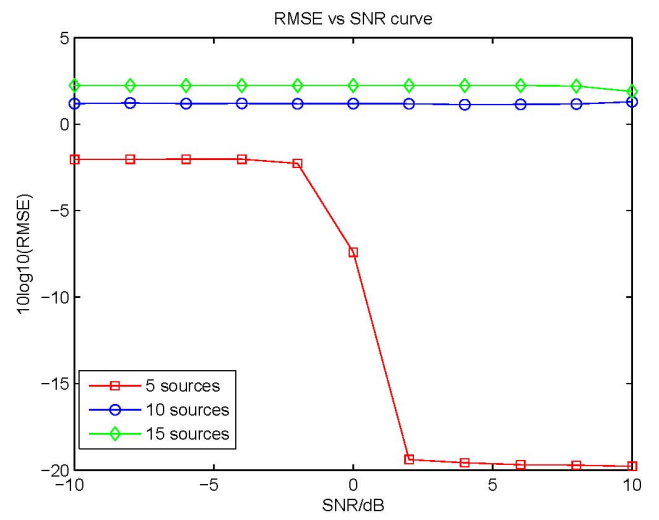

Fig. 13. RMSE of multiple sources under different SNR values

and 15 sources. The structure can distinguish the five sources accurately, and when the number of source increases to 10 , the performance degrades, but the structure can still figure out their locations with some errors. However, when 15 sources were applied, several fake peaks came out, and the estimation has such huge errors that the RMSE cannot vary from the change of SNR as Fig. 13 indicates.

\subsection{Dependency of error on direction of source}

As for multiple spherical array, all those spheres are placed on a plane, this kind of distribution may affect the estimation of sources which are defined within the plane. To figure out the dependency of error on the pitch direction of sources, we designed two other multiple arrays simulations. Structure 4 in subsection 5.3 was used, and the directions of the sources are $\left(30^{\circ}, 90^{\circ}\right),\left(90^{\circ}, 90^{\circ}\right)$, $\left(150^{\circ}, 90^{\circ}\right),\left(180^{\circ}, 90^{\circ}\right),\left(240^{\circ}, 90^{\circ}\right),\left(300^{\circ}, 90^{\circ}\right)$. The estimation results were shown in Fig. 14. In another case, the azimuth angle of the source was fixed at $60^{\circ}$, its pitch angle changes from $0^{\circ}$ to $180^{\circ}$ with step of $10^{\circ}$. Fig. 15 shows the RMSE of estimation under different SNR values. When $\phi=90^{\circ}$, the RMSE reaches its peak, indicates that the performance will degrades when sources were placed around the plane on which the multiple arrays were

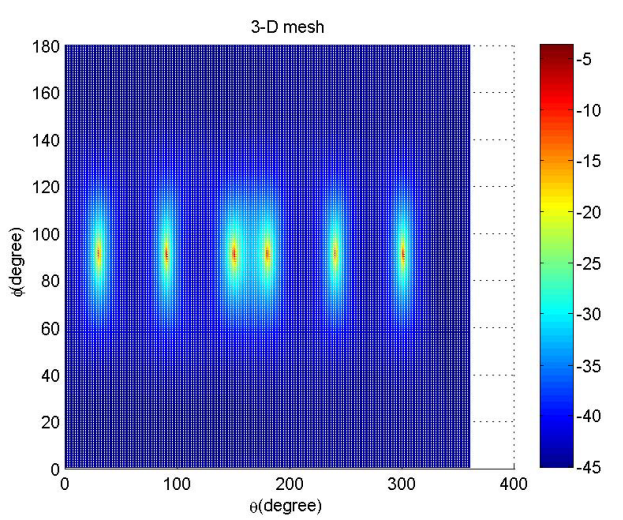

Fig. 14. Dependency of error under different SNR 


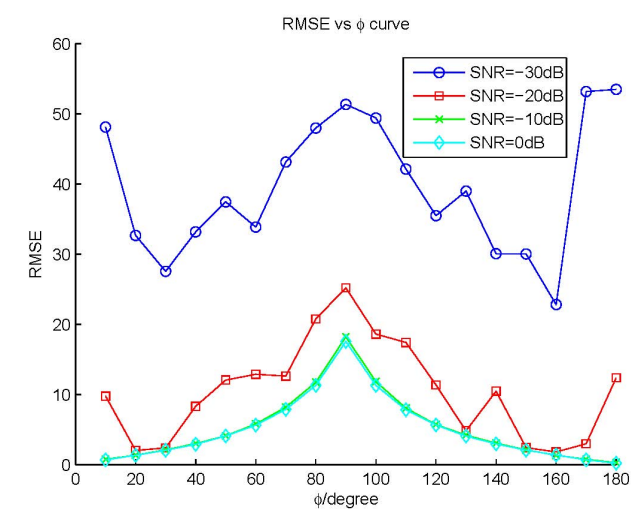

Fig. 15. DOA estimation of multiple sources when

distributed.

\section{Conclusion}

A multiple spherical arrays structure is developed for estimating the directions of multiple sources based on spherical harmonic decomposition of the sound field. The structure has following characteristics: (1) multiple spheres are placed as a circle on a plane, (2) on each sphere, the microphones are distributed uniformly as the usual single spherical array. Simulation results show that the multiple spherical arrays can deal with multiple sources more accurately by using less microphones on each sphere, while single spherical array in the same arrangement of microphones cannot provide the accurate results unless the number of microphones increase. Besides, the multiple spherical arrays can be applied to distinguish adjacent sources with high accuracy. Simulations verify the new structure's efficiency.

\section{References}

[1] C. Knapp and G. Carter, "The generalized correlation method for estimation of time delay," IEEE Transaction on Acoustic, Speech and Signal Processing, vol. 24, pp. 320-327, Aug. 1976.

[2] J. Benesty, "Adaptive eigenvalue decomposition algorithm for passive acoustic source localization," Journal of the Acoustical Society of America, vol. 107, pp. 384-391, Jan. 2000.

[3] H. Teutsch and W. Kellermann, "Eb-esprit: 2d localization of multiple wideband acoustic sources using eigen-beams," in ICASSP IEEE International Conference on Acoustic Speech and Signal Processing, Philadelphia, PA, United states, Mar. 2005, pp. 89-92.

[4] R. O. Schmidt, "Multiple emitter location and signal parameter estimation," IEEE Transactions on Antennas and Propagation, vol. 3, pp. 276-280, Mar. 1986.

[5] A. R. Leyman and T. S. Durrani, "Signal subspace technique for doa estimation using higher order statistics," in ICASSP IEEE International Conference on Acoustic Speech and Signal Processing, Detroit, MI, USA, May 1995, pp. 1956-1959.

[6] M. L. McCloud and L. L. Scharf, "A new subspace identification algorithm for high-resolution doa estimation," IEEE Transactions on Antennas and Propagation, vol. 50, pp. 1382-1390, Oct. 2002.

[7] R. Roy and T. Kailath, "Esprit-estimation of signal parameters via rotational invariance techniques," IEEE Transaction on Acoustic, Speech and Signal Processing, vol. 37, pp. 984-995, Jul. 1989.

[8] J. S. McGarrity, J. J. Soraghan, and T. S. Durrani, “A fast implementation of the esprit algorithm," in ICASSP IEEE International Conference on Acoustic Speech and Signal Processing, Albuquerque, New Mexico, USA, Apr. 1990, pp. 1001-1004.

[9] R. Hamza and K. Buckley, "Resolution enhanced esprit," IEEE Transactions on Signal Processing, vol. 42, pp. 688-691, Mar. 1994.

[10] H. Wang and M. Kaveh, "Coherent signal-subspace processing for the detection and estimation of angles of arrival of multiple wideband sources," IEEE Transaction on Acoustic, Speech and Signal Processing, vol. 33, pp. 823-831, Aug. 1985.

[11] J. E. F. del Rio and M. F. Catedra-Perez, "A comparison between matrix pencil and root-music for direction-of-arrival estimation making use of uniform linear arays,” Digital Signal Processing(USA), vol. 7, pp. 153-162, Jul. 1997.

[12] H. Teutsch and W. Kellermann, "Acoustic source detection and localization based on wavefield decomposition using circular microphone arrays," Journal of the Acoustical Society of America, vol. 120, pp. 2724-2736, Nov. 2006.

[13] B. Rafaely, "Analysis and design of spherical microphone arrays," IEEE Transaction on audio, speech and language processing, vol. 13, pp. 135-143, Jan. 2005.

[14] I. Balmages and B. Rafaely, "Open-sphere designs for spherical microphone arrays," IEEE Transaction on audio, speech and language processing, vol. 15, pp. 727-732, Feb. 2007.

[15] T. Noohi, N. Epain, and C. T. Jin, "Direction of arrival estimation for spherical microphone arrays by combination of independent component analysis and sparse recovery," in ICASSP IEEE International Conference on Acoustic Speech and Signal Processing, Vancouver, BC, Canada, May 2013, pp. 346-349.

[16] H. Teutsch and W. Kellermann, "Detection and localization of multiple wideband acoustic sources based on wavefield decomposition using spherical apertures," in ICASSP IEEE International Conference on Acoustic Speech and Signal Processing, Las Vegas, NV, United states, Mar. 2008, pp. 5276- 
5279.

[17] S. Argentieri, P. Danes, and P. Soueres, "Modal analysis based beamforming for nearfield or farfield speaker localization in robotics," in IEEE International Conference on Intelligent and Robot System, Beijing, China, Oct. 2006, pp. 866-871.

[18] T. D. Abhayapala and A. Gupta, "Spherical harmonic analysis of wavefields using multiple circular sensor arrays," IEEE Transaction on audio, speech and language processing, vol. 18, pp. 1655-1666, Aug. 2010.

[19] S. Kunis and D. Potts, "Fast spherical fourier algorithms," Journal of Computational and Applied Mathematics, vol. 161, pp. 75-98, Dec. 2003.

[20] H. Teutsch and W. Kellermann, "Acoustic source detection and localization based on wavefield decomposition using circular microphone arrays," Journal of the Acoustical Society of America, vol. 120, pp. 2724-2736, Nov. 2006.

[21] X. Li, S. F. Yan, X. C. Ma, and C. H. Hou, "Spherical harmonics music versus conventional music," Applied Acoustic, vol. 72, pp. 646-652, 2011.

[22] X. Mestre and M. A. Lagunas, "Modified subspace algorithms for doa estimation with large arrays," IEEE Transactions on Signal Processing, vol. 56, pp. 598-614, Feb. 2008.

[23] A. Gupta and T. D. Abhayapala, "Three-dimensional sound field reproduction using multiple circular loudspeaker arrays," IEEE Transaction on audio, speech and language processing, vol. 19, pp. 11491159, Jul. 2011.

[24] P.K.T. Wu, N. Epain, and C.T. Jin, “A dereverberation algorithm for spherical microphone arrays using compressed sensing techniques," in ICASSP IEEE International Conference on Acoustic Speech and Signal Processing, Kyoto, Japan, Mar. 2012, pp. 4053-4056.

[25] H. H. Chen and S. C. Chan, "Adaptive beamforming and doa estimation using uniform concentric spherical arrays with frequency invariant characteristics," Journal of VLSI Signal Processing Systems for Signal, Image, and Video Technology, vol. 46, pp. 15-34, Jan. 2007.

[26] Q. H. Huang and T. Song, "Doa estimation of mixed near-field and far-field sources using spherical array," in IEEE 11th International Conference on Signal Processing, Beijing, China, Oct. 2012, pp. 382-385.

[27] C.-I. C. Nilsen, I. Hafizovic, and S. Holm, "Robust 3$\mathrm{d}$ sound source localization using spherical microphone arrays," in 134th Audio Engineering Society Convention, Rome, Italy, May 2013, pp. 570-576.

[28] Y. Peled and B. Rafaely, "Linearly-constrained minimum-variance method for spherical microphone arrays based on plane-wave decomposition of the sound field," IEEE Transaction on audio, speech and language processing, vol. 21, pp. 2532-2540, Dec.
2013.

[29] D. B. Ward and T. D. Abhayapala, "Theory and design of higher order sound field microphones using spherical microphone array," in ICASSP IEEE International Conference on Acoustic Speech and Signal Processing, Orlando, FL, United states, May 2002, pp. 1949-1952.

[30] J. Meyer and G. Elko, "A highly scalable spherical microphone array based on a orthonormal decomposition of the soundfield," in ICASSP IEEE International Conference on Acoustic Speech and Signal Processing, Orlando, FL, United states, May 2002, pp. 1781-1784.

[31] T. S. Pollock, T. D. Abhayapala, and R. A. Kennedy, "Characterization of $3 \mathrm{~d}$ spatial wireless channels," in IEEE 58th Vehicular Technology Conference, Orlando, FL, United states, Oct. 2003, pp. 123-127.

[32] E. G. Williams, Fourier acoustic sound radiation and near field acoustical holography. Academic press, 1999.

[33] H. Teutsch, Modal Array Signal Processing: Principles and Applications of Acoustic Wavefield Decomposition. Springer, 2007.

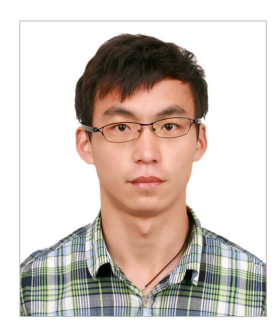

Fangzhou Wang $\mathrm{He}$ received B.S degree and M.S degree in electrical engineering from Beijing Institute of Technology, China, in 2012 and 2015, respectively. He is currently pursuing the Ph.D. degree in electrical engineering with Stevens Institute of Technology, Hoboken, NJ, USA. His research interests are statistical signal processing, optimization algorithm and passive sensing.

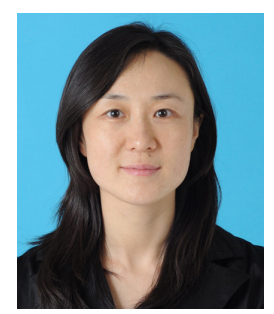

Xi Pan She received B.S., M.S. and $\mathrm{Ph} . \mathrm{D}$. degrees from Beijing Institute of Technology, China, in 1999, 2002 and 2007, respectively. She is an Associate Professor in the School of Mechatronical Engineering at Beijing Institute of Technology, China. She was a visiting scholar at Australian National University from 2011-2012, Her research interests include smart target detection, array signal processing, and acoustic signal processing. 